Running head: INFANTS' PROSPECTIVE CONTROL DURING OBJECT

MANIPULATION

Infants' prospective control during object manipulation in an uncertain environment

Janna M. Gottwald, Gustaf Gredebäck

Uppsala Child \& Baby Lab, Department of Psychology, Uppsala University, Sweden

This is the peer-reviewed version of the following article:

[Gottwald, J.M., \& Gredebäck, G. (2015). Infants' prospective control during object manipulation in an uncertain environment. Experimental Brain Research, 233, 2383-2390.]

The final publication is available at Springer via http://dx.doi.org/10.1007/s00221-015-4308-7.

Correspondence concerning this paper should be addressed to Janna Gottwald,

Department of Psychology, Uppsala University, Box 1225, 75142 Uppsala, Sweden.

E-mail: janna.gottwald@psyk.uu.se. 


\begin{abstract}
This study investigates how infants use visual and sensorimotor information to prospectively control their actions. We gave 14-month-olds two objects of different weight and observed how high they were lifted, using a Qualisys motion-capture system. In one condition the two objects were visually distinct (different color condition) in another they were visually identical (same color condition). Lifting amplitudes of the first movement unit were analyzed in order to assess prospective control. Results demonstrate that infants lifted a light object higher than a heavy object, especially when vision could be used to assess weight (different color condition). When being confronted with two visually identical objects of different weight (same color condition) infants showed a different lifting pattern than what could be observed in the different color condition, expressed by a significant interaction effect between object weight and color condition on lifting amplitude. These results indicate that a) visual information about object weight can be used to prospectively control lifting actions and that b) infants are able to prospectively control their lifting actions even without visual information about object weight. We argue that infants, in the absence of reliable visual information about object weight, heighten their dependence on non-visual information (tactile, sensorimotor memory) in order to estimate weight and pre-adjust their lifting actions in a prospective manner.
\end{abstract}




\section{Infants' prospective control during object manipulation in an uncertain environment}

A central aspect of action development is the acquisition of prospective control (von Hofsten 1993); the ability to adjust one's actions with respect to future task demands and action goals. In order to complete this forward planning we need to integrate information about the environment with current and future motor tasks (Ledouit, Casanova, Zaal and Bootsma 2013). Through prospective control we gain the ability to interact in a seamless manner with an uncertain, and ever changing, environment. To date, little is known about what type of information infants use to prospectively control their own goal-directed actions.

In order to investigate sensorimotor control processes that guide action, researchers often engage participants in tasks requiring them to lift objects of known and unknown weight. When lifting an object it is important to scale load-force according to the object's weight. To achieve this, multiple sources of information need to be integrated (Nowak, Glasauer and Hermsdörfer 2013). We know that adults rely on visual information (Buckingham, Cant and Goodale 2009; Buckingham and Goodale 2010a; Cole 2008; Gordon, Forssberg, Johansson and Westling 1991), sensorimotor memory (Buckingham and Goodale 2010b; Gordon, Westling, Cole and Johansson 1993; Mawase and Karniel 2010), and tactile information (Flanagan and Johansson 2009; Gordon, Forssberg, Johansson and Westling 1991; Johansson and Westling 1988) to prospectively control their lifting actions. All these sources of information are available prior to lift-off. For both visual information and sensorimotor memory (relying on perceived weight of the previously lifted object) this is selfevident, it is, however, interesting to note that tactile information about object weight can also be obtained prior to lift-off - within the brief period $(100-200 \mathrm{~ms})$ when the fingers grasp an object. Within this period, there is a large change in grip-force before an increase in loadforce that causes the object to overcome gravity and to move upwards (Johansson and Flanagan 2009; Johansson and Westling 1984). With all of this information, adults are able to 
provide a good estimate of weight and to prospectively control grip force according to the actual weight of the lifted object (Nowak et al. 2013).

Studies that have investigated prospective control of lifting actions in infancy have usually highlighted the importance of vision. Early work suggests that 2- but not 1-year-olds are able to prospectively control lifting actions based on weight (Forssberg, Kinoshita, Eliasson, Johansson, Westling and Gordon 1992). However, more recent work has demonstrated that infants can rely on visual information to estimate weight and to prospectively control lifting actions from a much earlier age. Nine-month-old infants are able to use color cues to estimate object weight and to prospectively scale their load force (Mash 2007). In this study, 9- to 15- month-olds were provided with light (10 g) and heavy (185 g) objects of different color, and lifting amplitudes were measured during the first $500 \mathrm{~ms}$ of lifting. On average, light objects were lifted higher than heavy objects. However, the actual lifting amplitude differed depending on prior experience: During test trials (where the color of light and heavy objects was switched) surprisingly light objects (using a color previously used to indicate heavy weight) were lifted higher (on average $11 \mathrm{~cm}$ ) than during baseline (on average $7 \mathrm{~cm}$ ), no such difference was observed during lifting of heavy objects (on average 8 $\mathrm{cm}$ in both baseline and test). This indication of prospective control is not unique for color but also applicable to other visually defined categories of objects, such as cones vs. bricks (Mash, Bornstein and Banerjee 2014). Prospective motor control has not only been demonstrated with respect to overt behavior but also with respect to neural correlates of motor planning (mu desynchronization; Nyström 2008). Marshall, Saby and Meltzoff (2013) demonstrated differential mu desynchronization for light and heavy objects both prior and during lifting. During reaching for light objects mu desynchronization was larger than during reaching for heavy objects, during lifting the reversed relation could be observed. Together these studies demonstrate that infants can rely on visual information to prospectively control actions, in this 
case, to scale load force to the perceived weight of objects (Marshall et al. 2013; Mash 2007; Mash, Bornstein and Banerjee 2014).

The degree to which infants are able to use non-visual sources of sensorimotor information to prospectively control their lifting actions is not known. This is unfortunate, as a richer understanding of the condition in which prospective motor control first emerges is needed. It provides useful clues to the ontogenetic origin of forward oriented control and inform us about the types of information that the developing action-perception system uses to interact with an uncertain world. The current study investigates how 14-month-old infants lift two objects of different weight (light and heavy) when acting in a certain (where vision is a reliable cue to object weight; here referred to as the different color condition) and in an uncertain environment (where the relation between color and weight changes over time; here referred to as the same color condition). Lifting amplitude of the initial part of each lifting action serves as measurement for prospective control. In order to better understand the temporal structure of prospective control we do not apply a fixed time window of analysis (similar to the $500 \mathrm{~ms}$ time window used by Mash 2007). Instead we detect the first movement unit (von Hofsten 1991; 2004) for each individual lift and analyze the amplitude of the upward movement at the end of this phase. In the context of prospective control, the current study is the first one to inspect lifting amplitudes with this level of precision. We focus on 14-months-olds, because infants at this age are able to use color cues to estimate the weight of objects (Mash 2007). We make the following prediction. (1) Based on Mash (2007) we expect infants in the different color condition to lift the light object higher than the heavy object. In addition we investigate if infants are able to prospectively control their lifting action in the absence of reliable visual information. Several possibilities exist.

(2a) If infants rely solely on visual information then they should fail to prospectively control their lifting action when visual cues are not present. In this case we expect infants in the same color condition to lift heavy objects lower and light objects higher than infants 
participating in the different color condition, see Figure 2 A. That is, applying the same load force to both objects in the same color condition will result in the light object being lifted higher and the heavy object being lifted lower. This alternative is here referred to as the visual hypothesis.

On the other hand, as demonstrated in adults, it is possible that infants use other sources of information to prospectively control their lifting actions. As for adults, it is possible that infants are able to rely either on tactile information during the brief period of contact with the object that precedes the enhanced load-force and lifting (Johansson and Flanagan 2009; Johansson and Westling 1984) or on sensorimotor memories (Buckingham and Goodale 2010b; Mawase and Karniel 2010) that maintain the weight of the previously lifted object. The availability of tactile information is ensured by having infants reach for cylinders that are narrow $(3.5 \mathrm{~cm})$ and tall $(10.1 \mathrm{~cm})$ facilitating translational movements of the object (that might be used to assess weight) during initial contact prior to lifting (see Figure 1). At the same time sensorimotor memories might play a role given that each weight was presented on several subsequent repetitions before the weight was changed (in a non-predictable manner, see methods for more detail). Studies with adults have demonstrated that these memories are not long lasting and highly influenced by the preceding trial (Nowak et al. 2013). In this case the probability that the current weight and the prior weight is the same is high (78\%). This leads us to postulate two alternatives to the visual hypothesis.

(2b) If infants are able to use non-visual cues (tactile information or sensorimotor memory) equally well as vision to estimate weight, performance should be unaffected by the removal of relevant visual cues. In this case, infants should maintain the same lifting amplitudes in the same color condition as demonstrated in the different color condition (larger lifting amplitudes for light than heavy objects) as expressed by a main effect of object weight on lifting amplitude only. This alternative is here referred to as the non-visual hypothesis. For an illustration of this effect see Figure 2 B. 
Final, a more speculative alternative is possible. Studies have suggested that non-visual sensorimotor information is important for object manipulation and can be efficiently used in the absence of visual information (Klatzky, Lederman and Reed 1987; Morrongiello, Humphrey, Timney, Choi and Rocca 1994). It has been shown that adults' tactile object exploration is more elaborated in the absence of visual information (Abravanel 1973). Accordingly, it might be the case that the absence of visual information heightens infants' sensitivity to non-visual sensorimotor information (tactile information from initial contact with the object on the current trial or from sensorimotor memory about weight obtained during the prior trial). This will result in more load-force being applied to heavy objects, and less to light objects, that cannot be visually differentiated. This leads us to our final hypothesis. (2c) If infants pay more attention to non-visual sensorimotor information in the absence of visual cues then performance might be enhanced by the removal of relevant visual cues. In this case the heavy object would be lifted higher and the light object would be lifted lower in the same color condition than the different color condition, for an illustration of this interaction effect see Figure 2 C. Formulated differently, infants should increase their loadforce to heavy objects and decrease load-force to light objects in the absence of visual information. We refer to this alternative as the enhanced non-visual hypothesis.

\section{Materials and Method}

\section{Participants}

The final sample consisted of 3014 -month-old infants (age $M=431$ days, $S D=4.79$, 12 female), who were randomly assigned to two conditions: Same color condition (age $M=$ 432 days, $S D=5.00 ; 7$ female) and different color condition (age $M=429$ days, $S D=4.51 ; 5$ female). Additional infants were tested but excluded due to technical problems $(n=1)$, lack of compliance with inclusion criteria ( $\mathrm{n}=7$, see data analysis for details) or due to poor-quality motion-tracking data $(\mathrm{n}=11)$. 
Participants were recruited from the Lab's database of parents who expressed interest in participating in research studies with their child. For participation parents got a gift voucher about 100 Swedish Crowns $(\approx 12$ Euro).

\section{Stimuli}

Two pairs of cylindrical objects $(10.1 \mathrm{~cm}$ in height, $3.5 \mathrm{~cm}$ diameter) served as test objects (Figure 1). Their relative dimensions ensured the availability of tactile information directly after the initial contact. The objects differ in color (blue vs. yellow) and weight (54 g vs. $271 \mathrm{~g}$ ) and are decorated with ribbons similar to the stimuli used by Marshall et al. (2013). They produce the same rattling sound by shaking and have one reflective marker each for motion tracking attached to it.

\section{Procedure}

After a give-and-take game with toys for the purpose of warm-up and to ensure that the infant is able to perform these actions, the infant was placed on the caregivers lap. They sat at a table opposite of the experimenter. First the experimenter placed the heavy object (out of the certain set) in front of the right hand of the parent. The parent lifted the object up, shook it with demonstrative joy and gave it back to the experimenter. This was done two times to show the action to the infant.

Then the lifting task started (Figure 1). The experimenter demonstrated the object by saying, "Look! A toy! Do you want to have it?" and placed it in front of the infant's dominant hand (the hand which is used for eating as per parent report) by saying, "Do you want to lift it up and shake it?" The infant was verbally encouraged to lift and shake the object. The trial ended either when the infant finished the action or after ten seconds have passed. At that point, the experimenter held her open hand towards the infant and asked for the object. If the infant was not giving it back, the experimenter delicately took it. This was done for 24 trials 
sub-divided into 3 blocks (each containing 8 trials). Every block consisted of $4( \pm 1)$ trials with the heavy object in the beginning and further $4( \pm 1)$ trials with the light object.

There were two conditions. In the same color condition either both blue or yellow objects were used (colors were counterbalanced). In the different color condition a blue and a yellow object were used (color-weight combination was counterbalanced). In both conditions one object was light and the other object was heavy.

\section{Data recording}

Data were recorded with a motion-tracking device (Qualisys Motion Capture Systems, Gothenburg, Sweden) with a sampling rate of $240 \mathrm{~Hz}$. An 8-camera motion capture system was used to identify and track the motion of the reflective markers attached to the object $(0.6$ $\mathrm{cm}$ in diameter). Initially infants also had one marker attached to the hand (10 from the same color condition and 9 from the different color condition), these markers were subsequently removed in order to reduce the dropout rate due to infants' irritation $(6.06 \%$ with and none without the marker on the hand) and increase the number of usable trials. Additionally, the session was filmed by two video cameras from the side and from a bird's-eyes view.

\section{Data analysis}

Videos were coded for different kind of movements (lifting, throwing, rolling, shifting, pushing, shaking) and handedness. Only the lifts of the child's dominant hand that were followed by a shaking movement were included, to rule out differential effects of handedness or prospective aspects of goal planning (reaching with the intention to place an object results in different movement patterns than reaching aimed towards an object that is intended to be thrown away; Claxton, Keen, and McCarty 2003). Infants who accomplished at least half of the procedure (12 trials) and delivered useable data of at least 2 consecutive lifts 
with different weighted objects were included in data analyses. On average, infants contributed 7 out of 12 trials per object, resulting in 14 out of 24 trials in total.

Vertical position and derived vertical velocity profiles were displayed for every single child using a custom Matlab script. The first movement unit started at the onset of the object's upwards movement, lasting one acceleration and deceleration phase of the velocity profile (von Hofsten 1991; 2004), in the vertical dimension. Lifting amplitudes at the end of the first movement unit were manually detected based on velocity profiles derived from vertical position data of the marker on the object (Figure 3). The force applied during this initial part of each lifting action, operationalized as the amplitude of the object (similar to Mash 2007) at the end of this initial upward movement, reflects prospective control whereas subsequent movement units involve corrective components derived from sensorimotor feedback and error signals in the internal forward model. To further assess force we causally inferred load force during the first movement unit using the following formula: force $[\mathrm{N}]=$ mass $[\mathrm{kg}] *$ acceleration $\left[\mathrm{meter} / \mathrm{sec}^{2}\right]$, while assuming a parabola-shaped lifting movement, amplitude $[$ meter $]=1 / 2$ acceleration $\left.\left[\mathrm{meter} / \mathrm{sec}^{2}\right] * \operatorname{time}_{\left[\sec ^{2}\right]}\right]$ or generally spoken $\mathrm{y}(\mathrm{t})=1 / 2 \mathrm{at}^{2}+\mathrm{y}_{0}$. Including the force of gravity, an estimation of the force the infants are applying to lift the object upwards results in the following formula: Force $=$ Force $_{\text {lift }}+$ Force $_{\text {gravity }}$, assuming constant force during this phase and no force loss during the movement.

In order to test the three hypotheses, average lifting amplitudes related to both weights were calculated for every participant, resulting in two values per participant. A 2x2 repeated measurement analysis of variance (ANOVA) with weight (within-subject variable) and condition (between-subject variable) for lifting amplitude at the end of the first movement unit was conducted. Additionally, two $t$-tests were conducted to further investigate the characteristics of the interaction between object weight and condition on lifting amplitude. A multiple comparison correction proposed by Benjamini and Hochberg (1995) was applied. 


\section{Results}

A main effect of object weight demonstrated that infants lifted light objects, $M D$ $($ mean difference $)=11.69 \mathrm{~cm}(S D=3.96)$, higher than heavy objects, $M D=8.33 \mathrm{~cm}(S D=$ 3.21), within the first movement unit, $F(1,28)=21.56, p<.001, \eta 2=.44$. In addition, the ANOVA demonstrated a significant interaction between weight and condition, $F(1,28)=$ $5.76, p=.023, \eta 2=.17$ (Figure $2 \mathrm{D}$ ), indicating a different lifting pattern in the first movement unit for both conditions. This suggests a rejection of the non-visual hypothesis and speaks instead - as performance is not the same for both conditions - for either the visual hypothesis or the enhanced non-visual hypothesis. The direction of the interaction effect is consistent with the enhanced non-visual hypothesis, see Figure 2.

Multi comparison corrected $t$-tests additionally revealed that the infants in the same color condition lifted heavy objects marginal significantly higher as compared to infants in the different color condition, $M D=2.12 \mathrm{~cm}(S D=1.23), t(28)=1.89, p=.069$ (two-tailed), favoring the enhanced non-visual hypothesis, but that there was no significant difference concerning light objects, $t(28)=0.94, p=.357$ (two-tailed).

The cautious inference of load force indicated that infants applied different amounts of force to heavy and light objects in both, the same color condition, force heavy $=2.82 \mathrm{~N}(S D=$ $0.11)$ and force light $=0.57 \mathrm{~N}(S D=0.02)$, and the different color condition, force heavy $=2.85 \mathrm{~N}$ $(S D=0.13)$ and forcelight $=0.59 \mathrm{~N}(S D=0.04)$

\section{Discussion}

This study investigates the use of sensorimotor information for prospective control during object manipulation in an uncertain environment (where vision does not provide reliable cues to weight). What we found was the following: When prior visual information on weight was available (different color condition), infants lifted light objects higher than heavy 
objects, in accordance with our first prediction. Infants are able to prospectively control their lifting action and to apply a different amount of force to lift objects that are clearly heavy or light.

During the same color condition, in which visual information could not be used to assess object weight, infants showed a different lifting pattern than what was observed in the different color condition. This significant interaction does not correspond with the visual hypothesis (suggesting a larger difference between heavy and light object than what was observed in the different color condition) or with the non-visual hypothesis (suggesting the same lifting amplitudes for both conditions).

The rejected non-visual hypothesis states that infants could complete the task equally well in the absence of vision whereas the supported enhanced non-visual hypothesis states that infants become more sensitive to the actual weight when visual cues are removed. That is, lifting heavy objects higher and light objects lower in the same color condition than the different color condition. Infants seem better able to adjust their load-force in accordance with the actual weight of the object when they cannot rely on visual information. From this we can conclude that infants are able to prospectively control their actions and to scale their loadforce according to the weight of objects even in the absence of visual information differentiating heavy from light objects. This cautiously suggests that the absence of visual information may heighten infants' sensitivity to other forms of information that provide more reliable cues about the actual weight of the to-be lifted object.

As stated above, there are two primary sources of non-visual information that might be used to prospectively control actions. Infants might rely on tactile information and perceive the weight of the object proprioceptively prior to lifting (during a period of enhanced grip force prior to lifting; Johansson and Flanagan 2009; Gordon et al. 1991; Johansson and Westling 1988a). Another option is that infants maintain a sensorimotor memory based on the 
preceding lift to make a fair assumption about the current weight (Buckingham and Goodale 2010b; Gordon et al. 1993; Mawase and Karniel 2010). These options are not mutually exclusive and more research is needed to investigate if infants solely rely on one of these or on a combination of the two.

That infants might perform more efficiently in the absence of reliable visual information on object weight becomes highly interesting in light of findings demonstrating a dominance of visual information over other sources of information (e.g., Cole 2008; Buckingham and Goodale 2010b; Forssberg et al. 1992; Gordon et al. 1991) and of the repeated demonstration that infants also rely on vision to estimate object weight (Marshall et al. 2013; Mash 2007; Mash et al. 2014). It has been shown that adults usually rely on vision during object exploration, but that their tactile exploration behavior is more elaborated in the case of absent visual information (Abravanel 1973). In line with this finding, Klatzky et al. (1987) argue against the dominance of vision, stating that the tactile system provides a similar rich amount of information as the visual system. Morrongiello et al. (1994) compared how blind and blindfolded children with typical vision (aged from 3 to 8 years) explore objects, demonstrating that both groups do not differ in their performance, indicating that previous visual experience is not essential for tactile object manipulation. Together this research shows that sensorimotor information is important for object manipulation and can be efficiently used in the absence of visual information. The current study demonstrates that sensorimotor information can be used by infants to assess object weight and prospectively control lifting.

One alternative interpretation of the results might be that infants apply a default strategy in an uncertain environment, that is, applying the same force to both heavy and light objects when color is no reliable cue for object weight. This would inevitably result in lower lifts for heavy than for light objects. One way to test this alternative suggestion is to cautiously infer load-force using a force calculation while assuming constant acceleration and 
no force loss during the movement, as stated in the methods and results sections. According to this estimation infants applied different amounts of force to heavy and light objects in both conditions. Based on this, it seems unlikely that infants simply apply a default strategy, further strengthening the suggestion that infants rely on non-visual cues and that their performance might even be enhanced in the absence of reliable visual information.

Some caveats should be noted. First of all, a substantial number of infants had to be excluded from the analysis (39\%). This is often the case with infancy research. Depending on the measurement and the experimental paradigm exclusion rates up to $50 \%$ are common in infancy research due to fussiness and habituation. Compared to adults, infants have a shorter attention span and less tolerance for experimental procedures. Secondly, due to the characteristics of infancy research outlined above, we opted for a design that ensured a large number of trials per condition. This included using a between-subject design with a semifixed presentation order (each block starting with heavy objects and switching over to light after a pre-determined number of trials). Last but not least, the significant interaction effect between condition and weight supports the enhanced non-visual hypothesis, however, one of the follow-up multiple comparison corrected t-tests did not reach trend-level significance. Consequently, our interpretation seems to apply especially for lifting of heavy objects and has to be treated more cautiously in the case of light objects. Future studies should address this research question using a large sample of infants, a within-subject design, and a randomized presentation order.

In summary, this work demonstrates that 14-month-olds use non-visual cues to prospectively control their object-related actions. In the absence of relevant visual information, infants might plan their actions even more efficient due to their heightened sensitivity to either tactile information or sensorimotor memory of weight. We conclude with stating that infants by the age of 14 month have an impressive ability to control their actions 
prospectively and that infants can rely on several different sources of information to assess object properties. By using the first movement unit of the lift instead of a fixed time criterion, we introduce a new measurement for investigating prospective motor control in the context of object manipulation. Future research has to separate out the unique contribution of both tactile information and sensorimotor memory and also to gain a better understanding of how these processes interact with visual information to provide a rich understanding of the world. 


\section{Acknowledgements}

This project has received funding from the European Union's Seventh Framework Programme for research, technological development and demonstration under grant agreement no 289404 and from ERC-StG CACTUS 312292. We thank Claes von Hofsten for fruitful theoretically discussions, Torsten Leitner for valuable input and discussions on the physical background of force calculation, Estefanía Domínguez Martínez for helpful support in Matlab programing, Mattias Stridbeck for illustrations, Marcus Lindskog for valuable input on probability calculations, and last but not least the members of the Uppsala Child and Baby Lab for constructive feedback on a previous version of the manuscript.

\section{Conflict of interest}

The authors declared that they had no conflicts of interest with respect to their authorship or the publication of this article.

\section{Compliance with Ethical Standards}

All procedures performed in studies involving human participants were in accordance with the ethical standards of the regional ethics committee and with the 1964 Helsinki declaration and its later amendments or comparable ethical standards. Informed consent was obtained from all individual participants included in the study. 


\section{References}

Abravanel E (1973) Division of labor between eye and hand when perceiving shape. Neuropsychology, 11, 207-211.

Benjamini Y \& Hochberg Y (1995) Controlling the false discovery rate: A practical and powerful approach to multiple testing. Journal of the Royal Statistical Society. Series B (Methodological), 57, 289-300.

Buckingham G, Cant JS, Goodale MA (2009) Living an a material world: How visual cues to material properties affect the way that we lift objects and perceive their weight. Journal of Neurophysiology, 102, 3111-3118.

Buckingham G, Goodale MA (2010a) Lifting without seeing: The role of vision in perceiving and acting upon the size weight illusion. PLoS one, 5, DOI:

10.1371/journal.pone.0009709.

Buckingham G, Goodale MA (2010b) The influence of competing perceptual and motor priors in the context of the size-weight illusion. Experimental Brain Research, 205, 283288.

Claxton LJ, Keen R, McCarty ME (2003) Evidence of motor planning in infant reaching behavior. Psychological Science, 14, 354-356.

Cole KJ (2008) Lifting a familiar object: Visual size analysis, not memory for object weight, scales lift force. Experimental Brain Research, 188, 551-557.

Forssberg H, Kinoshita H, Eliasson AC, Johansson RS, Westling G, Gordon AM (1992) Development of human precision grip. Anticipatory control of isometric forces targeted for object's weight. Experimental Brain Research. 90, 393-398.

Gordon AM, Forssberg H, Johansson RS, Westling G (1991) Visual size cues in the programming of manipulative forces during precision grip. Experimental Brain Research, 83, 477-482. 
Gordon AM, Westling G, Cole KJ, Johansson RS (1993) Memory representations underlying motor commands used during manipulation of common and novel objects. Journal of Neurophysiology, 69, 1790-1796.

Johansson RS, Westling G (1984) Roles of glabrous skin receptors and sensorimotor memory in automatic control of precision grip when lifting rougher or more slippery objects. Experimental Brain Research, 56, 550-564.

Johansson RS, Westling G (1988) Coordinated isometric muscle commands adequately and erroneously programmed for the weight during lifting task with precision grip. Experimental Brain Research, 71, 59-71.

Johansson RS, Flanagan JR (2009) Coding and use of tactile signals from the fingertips in object manipulation tasks. Nature Reviews Neuroscience, 10, 345-359.

Klatzky RL, Lederman S, Reed C (1987) There's more to touch than meets the eye: The salience of object attributes for hap tics with and without vision. Journal o Experimental Psychology: General, 116, 356-369.

Ledouit S, Casanova R, Zaal FTJM, Bootsma RJ (2013) Prospective control in catching: The persistent angle-of-approach effect in lateral interception. PLOS ONE, 8, DOI:10.1371/journal.pone.0080827.

Marshall PJ, Saby JN, Meltzoff A. (2013) Infant brain responses to object weight: Exploring goal-directed actions and self-experience. Infancy, 18, 942-960.

Mash C (2007) Object representation in infants' coordination of manipulative force. Infancy, $12,329-341$.

Mash C, Bornstein MH, Banerjee A (2014). Development of object control in the first year: emerging category discrimination and generalization in infants' adaptive selection of action. Developmental Psychology, 50, 325-335.

Mawase F, Karniel A (2010) Evidence for predictive control in lifting series of virtual objects. Experimental Brain Research, 203, 447-452. 
Morrongiello BA, Humphrey GK, Timney B, Choi J, Rocca PT (1994) Tactual object exploration and recognition in blind and sighted children. Perception, 23, 833-848.

Nowak DA, Glasauer S, Hermsdöfer J (2013) Force control in object manipulation - A model for the study of sensorimotor control strategies. Neuroscience and Biobehavioral Reviews, 37, 1578-1586

Nyström P (2008) The infant mirror neuron system studied with high density EEG. Social Neuroscience, 3, 334-347.

von Hofsten C (1991) Structural of early reaching movements. A longitudinal study. Journal of motor behavior, 23, 280-292.

von Hofsten C (1993) Prospective Control: A basic aspect of action development. Human Development, 36, 253-270.

von Hofsten C (2004) An action perspective on motor development. TRENDS in Cognitive Sciences, 8, 266-272. 
Figure 1. Picture of the four objects differing in color and weight (left picture) and two snapshots of a 14-month-old lifting one of the objects (middle and right picture). 
Figure 2. Hypothesized result patterns (A-C) and actual results (D) of the mean lifting amplitude of the first movement unit as a function of object weight (heavy, light) and experimental condition (dashed line: same color condition, solid line: different color condition). Hypotheses: A) The visual hypothesis states that infants in the same color condition lift heavy objects lower and light objects higher than infants in the different color condition, B) The non-visual hypothesis states that infants in the same color condition show the same lifting amplitudes as infants in the different color condition, C) The enhanced nonvisual hypothesis states that infants in the same color condition lift heavy objects higher and light objects lower than infants in the different color condition. Results: D) Mean lifting amplitudes (in $\mathrm{cm}$ ) of the first movement unit as a function of object weight and experimental condition. Error bars indicate the standard error of the mean. 
Figure 3. Position and velocity profiles for one typical lifting action of one participant. Movement units (mu) are defined based on the velocity profile. 